\title{
Commentary
}

Copyright (C) All rights are reserved by Mao Sheng Yang

\section{A Coin has Two Sides: Use Marijuana with Cautions}

\author{
Ting $\mathrm{Chu}^{1,2}$ and Mao Sheng Yang ${ }^{*}$ \\ ${ }^{1}$ Research Center of Translational Medicine, Jishou University School of Medicine, China \\ ${ }^{2}$ Graduate School of Education, Sehan University, Republic of Korea
}

*Corresponding author: Mao Sheng Yang, Professor, Laboratory of Disorders Genes and Department of Pharmacology, Research Center of Translational Medicine, Jishou University School of Medicine, 43 Wuling East Road, Da Tian Wan Campus, Jishou Hunan 416000, China.

Received Date: October 30, 2019

Published Date: November 06, 2019

\section{Commentary}

Whether used legally or illegally, the use of marijuana has a long history. However, the benefits and harms of medical use of marijuana and how to use marijuana have not yet reached consensus in the medical community [1,2]. Cavazos-Rehg PA, et al. [2] study suggests that most marijuana-related tweets support marijuana use and believe marijuana has health benefits and/or should be legalized [2]. Colorado passed Amendment 20 in November 2000, which supports the use marijuana by people who suffered one of the following eight "debilitating medical conditions": HIV/AIDS, glaucoma, severe nausea, severe pain, cancer, cachexia, seizures, and muscle spasms [3]. But, according to an anonymous webbased electronic survey and 520 family physicians (FPs) responses, forty-six percent of FPs does not support the recommended use of marijuana, and only nineteen percent of FPs believes that it should be recommended [3]. Despite the dramatic increase in medical use of marijuana by adults, pregnant women and adolescents, there are few clinical trials to demonstrating the benefits and harms of medical use of marijuana, and few trials under the auspices of the Food and Drug Administration (FDA) to compare medicinal marijuana with traditional analgesics [2-5]. Therefore, it is time to pay more attention to using marijuana with cautions, and shift our focus toward improving quality of life of patients by scientific and rational use of marijuana. Three arguments support the conclusion above.

Firstly, marijuana is increasingly available and used in many forms by people of different ages, genders and races. Hence, formulating relevant laws and regulations for the clinical application of marijuana, and scientific and rational use of marijuana under the guidance of physicians or pharmacists, are the best choice to reducing marijuana harms and improving the quality of life of patients $[1,2,5]$.

Secondly, the marijuana contains hundreds of pharmacologically active ingredients or components, and most of them have not been well characterized. The actions of marijuana and its mechanisms, clinical indications and contraindications remain to be further studied. By the way, marijuana from different sources or geographies may have different or uncertain effects, and may contain unknown contaminants $[1,5]$.

Finally, the growing literature or evidence supports the efficacies of medical marijuana use, especially for chronic pain, neuropathic pain, spasticity, nausea, vomiting, and cachexia that are unresponsive to mainstream treatments $[1,4,5]$, and supports the Colorado passed Amendment 20 in November 2000, and suggests that medical marijuana use may benefit for patients [2]. However, the adverse effects of medical marijuana use also should be concern, such as develop dependence, develop schizophrenia, develop depressive disorders, cognitive impairment, increase symptoms of obstructive lung disease and may promote cancer-cell proliferation $[1,3]$.

In one word, we are not the first one to support the medical marijuana use to relieve the suffering of patients. We suggest that rational using marijuana under the guidance of physicians or pharmacists, and scientific evaluating whether the medicinal benefits of marijuana outweigh the risks or potential harms may be more helpful to our patients.

\section{Acknowledgement}

None.

\section{Conflict of Interest}

Authors have disclosed no conflicts of interest.

\section{References}

1. Bostwick JM, Reisfield GM, DuPont RL (2013) Clinical decisions. Medicinal use of marijuana. N Engl J Med 368(9):866-868.

2. Cavazos-Rehg PA, Krauss M, Fisher SL, Salyer P, Grucza RA, et al. (2015) Twitter chatter about marijuana. J Adolesc Health 56(2): 139-145. 
3. Kondrad E, Reid A (2013) Colorado family physicians' attitudes toward medical marijuana. J Am Board Fam Med 26(1): 52-60.

4. Grant I, Atkinson JH, Gouaux B, Wilsey B (2012) Medical marijuana: clearing away the smoke. Open Neurol J 6: 18-25.
5. Hill KP (2015) Medical Marijuana for Treatment of Chronic Pain and Other Medical and Psychiatric Problems: A Clinical Review. JAMA 313(24) 2474-2483. 